\title{
Neural Mechanisms for Acoustic Signal Detection under Strong Masking in an Insect
}

\author{
- Konstantinos Kostarakos and Heiner Römer \\ Institute of Zoology, Karl-Franzens-University, $8010 \mathrm{Graz}$, Austria
}

Communication is fundamental for our understanding of behavior. In the acoustic modality, natural scenes for communication in humans and animals are often very noisy, decreasing the chances for signal detection and discrimination. We investigated the mechanisms enabling selective hearing under natural noisy conditions for auditory receptors and interneurons of an insect. In the studied katydid Mecopoda elongata species-specific calling songs (chirps) are strongly masked by signals of another species, both communicating in sympatry. The spectral properties of the two signals are similar and differ only in a small frequency band at $2 \mathrm{kHz}$ present in the chirping species. Receptors sharply tuned to $2 \mathrm{kHz}$ are completely unaffected by the masking signal of the other species, whereas receptors tuned to higher audio and ultrasonic frequencies show complete masking. Intracellular recordings of identified interneurons revealed two mechanisms providing response selectivity to the chirp. (1) Response selectivity is when several identified interneurons exhibit remarkably selective responses to the chirps, even at signal-to-noise ratios of $-21 \mathrm{~dB}$, since they are sharply tuned to $2 \mathrm{kHz}$. Their dendritic arborizations indicate selective connectivity with low-frequency receptors tuned to $2 \mathrm{kHz}$. (2) Novelty detection is when a second group of interneurons is broadly tuned but, because of strong stimulus-specific adaptation to the masker spectrum and "novelty detection" to the $2 \mathrm{kHz}$ band present only in the conspecific signal, these interneurons start to respond selectively to the chirp shortly after the onset of the continuous masker. Both mechanisms provide the sensory basis for hearing at unfavorable signal-to-noise ratios.

Key words: acoustic masking; frequency tuning; insects; novelty detection; selective coding

\section{Significance Statement}

Animal and human acoustic communication may suffer from the same "cocktail party problem," when communication happens in noisy social groups. We address solutions for this problem in a model system of two katydids, where one species produces an extremely noisy sound, yet the second species still detects its own song. Using intracellular recording techniques we identified two neural mechanisms underlying the surprising behavioral signal detection at the level of single identified interneurons. These neural mechanisms for signal detection are likely to be important for other sensory modalities as well, where noise in the communication channel creates similar problems. Also, they may be used for the development of algorithms for the filtering of specific signals in technical microphones or hearing aids.

\section{Introduction}

Communication is fundamental for our understanding of animal and human behavior. It requires the encoding of information in a

\footnotetext{
Received March 9, 2015; revised May 28, 2015; accepted June 16, 2015.

Author contributions: K.K. and H.R. designed research; K.K. performed research; K.K. analyzed data; K.K. and H.R. wrote the paper.

Research was funded by the "Austrian Science Foundation" (FWF), Project P21808-B09 and P23896-B24. We thank Manfred Hartbauer for experimental support and fruitful discussions and Berthold Hedwig for his critical comments on an earlier draft of this manuscript.

The authors declare no competing financial interests.

This article is freely available online through the J Neurosci Author Open Choice option.

Correspondence should be addressed to Konstantino Kostarakos, Institute of Zoology, Karl-Franzens-University, Universitaetsplatz 2, 8010 Graz, Austria. E-mail: konstantinos.kostarakos@uni-graz.at.

DOI:10.1523/JNEUROSCI.0913-15. 2015

Copyright $@ 2015$ Kostarakos and Römer

This is an Open Access article distributed under the terms of the Creative Commons Attribution License Creative Commons Attribution 4.0 International, which permits unrestricted use, distribution and reproduction in any medium provided that the original work is properly attributed.
}

signal by a sender, which is transmitted via a transmission channel and detected by a receiver (Bradbury and Vehrenkamp, 2011). However, the acoustic environment of many vocalizing species is complex, as it contains a number of unknown sound sources of the same and different species. Signaling in such groups poses problems for receivers to detect and classify signals (Hulse, 2002; Brumm and Slabbekoorn, 2005; Wiley, 2006, 2013). For humans the "cocktail party problem" (Cherry, 1953) refers to the difficulty of understanding speech in noisy social settings (Yost, 1997; Bronkhorst, 2000). Despite this difficulty, humans and other species can successfully listen and orient to individual sound sources, using a process termed auditory scene analysis (Bregman, 1990). Animal acoustic communication may suffer from similar problems (Bee and Micheyl, 2008), because background noise in choruses of calling individuals can be dramatic. In species-rich tropical rainforests, $>50$ species of insects 
and frogs create an acoustic background with vocalizations at high sound pressure levels (Römer et al., 2010; Schwartz and Bee, 2013). Research in the past decade has demonstrated that the selection pressure from the masking of background noise has shaped evolutionary adaptations that allow both senders and receivers to cope with noise (Brumm, 2013). These include individual adjustments of signal properties such as increased loudness (Lombard effect), signal duration and/or redundancy, sound frequency separation, signal timing, or the use of multimodal signals (Higham and Hebets, 2013). Other adaptations may involve the reception and neural processing of sound by the peripheral or central nervous systems, such as narrow frequency filtering, spatial release from masking, or gain control mechanisms (Römer, 2013). Although behavioral studies in various taxa indicate reliable communication at remarkably low signal-to-noise ratios (SNRs), the underlying neuronal mechanisms are often unclear.

Here we studied these mechanisms in two closely related species of acoustic insects that live in sympatry in the tropical rainforest. Both species of katydid belong to the Mecopoda elongata complex. The calling song of the "chirper" consists of broadband chirps that are repeated every $2 \mathrm{~s}$. Males of the competing species produce continuous trills of $>105 \mathrm{~dB}$ SPL (Krobath, 2013). The song of the "triller" represents a strong external noise that should mask the neural representation of the chirp in receivers. However, contrary to the expectation from theory and from empirical cases with other species using broadband signals (Greenfield, 1988; Römer et al., 1988), acoustic communication of the chirper appears to be almost unaffected by the continuous and loud song of the trilling species despite similar broadband power spectra of both signals (Siegert et al., 2013). A narrow $2 \mathrm{kHz}$ bandwidth in the spectrum of the chirp provides the essential cue for unmasking. The sound energy of this $2 \mathrm{kHz}$ bandwidth is $\sim 25 \mathrm{~dB}$ higher in the conspecific chirp compared with the masking trill. Behavioral studies revealed that males of the chirper synchronize their songs even at SNRs of $-8 \mathrm{~dB}$. However, the behavioral response breaks down if the $2 \mathrm{kHz}$ component in the chirps is equalized to the level of the masker (Siegert et al., 2013).

Here, we present two neural mechanisms for selective responses to the song provided by different identified auditory interneurons. These mechanisms are shaped by exploiting the activity of receptors that are tuned to the $2 \mathrm{kHz}$ component of the chirp: (1) selective tuning where some interneurons are selectively tuned to this low-frequency component and respond only to conspecific chirps and (2) novelty detection where broadly tuned interneurons use a mechanism called "novelty detection" (Schul et al., 2012) in which strong adaptation in response to the continuous trill ensures a selective response to the frequency "novelty" in the chirp. The responses of these specific interneurons sufficiently explain the reliable signal detection observed in behavior, even at very low SNRs.

\section{Materials and Methods}

Animals. Males and females of M. elongata (Ensifera, Tettigoniidae, and Mecopodini) were used for neurophysiology. Chirps of the chirper are identical to those of "species S" (Sismondo, 1990), and songs of the trilling species are identical to those of "Mecopoda sp. 2" (Korsunovskaya, 2008). There was no difference observed in the structure and response properties of auditory interneurons between males and females. Insects were taken from a colony at the University of Graz that was originally established with individuals collected in Malaysia close to the field station Ulu Gombak near Kuala Lumpur. The insects were reared in a $12 \mathrm{~h} \mathrm{light/dark}$ cycle at a temperature of $27^{\circ} \mathrm{C}$ and $70 \%$ relative humidity. They were fed with fresh lettuce, oat flakes, and fish food ad libitum.
Acoustic stimulation. Calling songs of both species were recorded from isolated males at a distance of $15 \mathrm{~cm}$ to the signaling individual using a calibrated, free-field condenser microphone (type 40AC; G.R.A.S. Sound \& Vibration A/S) with a flat frequency response between $10 \mathrm{~Hz}$ and 40 $\mathrm{kHz}$. The microphone output was amplified using a preamplifier (type 26AM; G.R.A.S. Sound \& Vibration A/S) and a power module (type 12AK; G.R.A.S. Sound \& Vibration A/S). A/D conversion was performed via an external audio interface (Edirol FA-101; Roland) operating at a sampling rate of $96 \mathrm{kHz}$.

The chirp used for playback consisted of 15 syllables (chirp duration $285 \mathrm{~ms}$, syllable period $20 \mathrm{~ms}$ ) with gradually increasing amplitude and was repeated at a rate of $0.4 \mathrm{~Hz}$. The trill of the competing Mecopoda species consisted of a soft syllable followed by two syllables of high amplitude, a stereotypical pattern that was repeated with a period duration of $30 \mathrm{~ms}$. Playback of sound signals was controlled in Cool Edit Pro 2.0 driving an Edirol A/D audio interface operating at a sampling rate of $96 \mathrm{kHz}$. Sound signals were attenuated (PA-5; Tucker-Davis Technologies) and amplified using an amplifier with a flat frequency response up to $100 \mathrm{kHz}$ (NAD 214; NAD Electronics). Signals were broadcast by two ultrasonic magnetic speakers (MF1-S) with flat frequency characteristics from 1 to $>40 \mathrm{kHz}$ (Tucker-Davis Technologies). The spectral composition of both the chirp and the trill at the position of the preparation is shown in Figure $1 D$. Because of the reduced sampling rate of the Edirol A/D audio interface, the signal playback resulted in a strong attenuation of frequencies $>40 \mathrm{kHz}$. We thus simulated the frequency spectrum of a signal as being perceived at medium sender-receiver distances, where ultrasonic frequencies are strongly attenuated (Keuper et al., 1986; Romer and Lewald, 1992).

Pure tone sound pulses were generated with Cool Edit Pro 2.0 at a sampling rate of $192 \mathrm{kHz}$. The speakers were positioned next to each other at one side of the longitudinal body axis of the preparation at a distance of $15 \mathrm{~cm}$. Sound pressure levels at the preparation were calibrated at the position of the ears using a $1 / 2$ inch microphone (type 2540; Larson Davis) connected to a sound level meter (CEL 414; Casella CEL). The sound calibration was performed by presenting a continuous loop of the three last syllables in the chirp.

The chirps and masking trill were broadcast either separately or simultaneously at different SPLs. The variation of SNRs usually started with an SNR of $0 \mathrm{~dB}$ with $75 \mathrm{~dB}$ SPL for both signals, and was decreased in $3 \mathrm{~dB}$ steps by attenuating the intensity of the chirp. The tuning of acoustic neurons was studied with iso-intensity response profiles. In contrast to conventional tuning curves using threshold values at various frequencies, such iso-intensity response profiles provide tuning information at suprathreshold or more relevant intensity values. Tuning was studied using sound pulses (duration $50 \mathrm{~ms}$ ) of different frequency $(1,2,5,10,15,20$, 30,40 , and $55 \mathrm{kHz}$ ) broadcast at $80 \mathrm{~dB}$ SPL. To study the impact of the low-frequency components of the chirp on signal discrimination under the masking trill we also used modified chirps as acoustic stimuli. For the experimental approach, the frequency band at $2 \mathrm{kHz}$ was attenuated to the same level as in the spectrum of the trill, using the FFT filter function provided by Cool Edit Pro 2.0.

Intracellular recordings. Intracellular recordings were performed from auditory interneurons within the prothoracic ganglion. Animals were anesthetized with chlorethyl and the antennae and middle and hind legs were removed. The animals were fixed ventral side up on a holder by using dental wax. The head was slightly tilted backward and fixed on the holder while the tarsi of the forelegs were waxed onto thin wires. An incision between abdomen and thorax reduced hemolymphe pressure and tissue movements from breathing. The prosternum was removed to expose the prothoracic ganglion and the perineurium was carefully removed in the vicinity of the auditory neuropil to allow a smooth penetration of the microelectrode. The prothoracic ganglion was covered with insect saline. The preparation was then placed at a distance of $15 \mathrm{~cm}$ to the speakers. The ganglion was stabilized between a small metal platform at its dorsal side and a metal ring at its ventral side with the platform serving as a reference electrode for intracellular recordings. Microelectrodes were filled with 5\% Lucifer yellow $\mathrm{CH}$ (Sigma-Aldrich) dissolved in aqua destillata or with $0.8 \%$ Alexa 555 and 568 hydrazide (Molecular 
Probes) dissolved in $0.2 \mathrm{M}$ lithium chloride for intracellular staining using conventional protocols.

Intracellular recordings were mainly performed within the anterior part of the prothoracic ganglion at the area of the auditory neuropil (Römer, 1983; Stolting and Stumpner, 1998). Neuronal responses were amplified by a BA-01X amplifier (npi electronic) in bridge mode. Fluorescent dyes were iontophoretically injected into the neurons by hyperpolarizing current injection (0.5-5 nA) for 5-30 min. After histological processing and clearing in methyl salicylate, a Zeiss Axioplan epifluorescence microscope (Carl Zeiss) was used to visualize the morphology of the neurons. Images were taken with an Axiocam ERc 5s (Carl Zeiss). Neurons were reconstructed manually from image stacks using ImageJ (NIH) and Photoshop CS6 (Adobe Systems) software. Neurons were identified according to their morphology and response patterns.

Terminology. The terminology of neurons was based on their frequency selectivity and axonal projections. The letters LF indicate lowfrequency neurons being sharply tuned to the 2 $\mathrm{kHz}$ component of the song. The letters BF indicate broadband neurons selective over a bandwidth of $>10 \mathrm{kHz}$. The third letters indicate axonal projections ascending toward the brain (A), descending toward the mesothoracic ganglion (D), and a T-shaped structure of axons both ascending and descending (T). Numbers were added to discriminate between neurons with similar anatomy and tuning properties but differing in other physiological properties.

Data recordings and analysis. All recording channels were digitized at $20.8 \mathrm{kHz}$ and 16-bit amplitude resolution with $0.153 \mathrm{mV}$ per increment using a CED 1401 micro3 data acquisition interface. Data were recorded to the hard disk of a PC using Spike 2 software (Cambridge Electronic Design). Neural recordings were analyzed off-line using Neuro-Lab software (Knepper and Hedwig, 1997). Responses were analyzed using peristimulus time histograms and averages of instantaneous spike frequency or postsynaptic membrane potentials.

To determine the amount of masking of the chirp response by the trill, the neural responses to the trill were averaged within a time window of $300 \mathrm{~ms}$ before the chirp and subtracted from the responses to the chirps presented simultaneously with the trill. This difference indicated the degree of neural representation of the masked chirp. In a similar way the neural response to unmasked chirps was calculated by subtracting spontaneous activity (again over $300 \mathrm{~ms}$ before the chirps) from the activity elicited by the chirp. The response to chirps (for both unmasked and masked conditions) was also calculated within a time window of $300 \mathrm{~ms}$ after stimulus onset, including $10 \mathrm{~ms}$ for the latency of receptor responses. The relative values of spike activity to the chirp during the trill (with the response to the chirp only set as 100\%) provide a quantitative measure for the response selectivity of all types of neurons.

Statistical evaluation was performed by using SigmaPlot 12.5 software (Systat). $T$ tests were used for normally distributed data and a MannWhitney rank sum test for data not normally distributed.

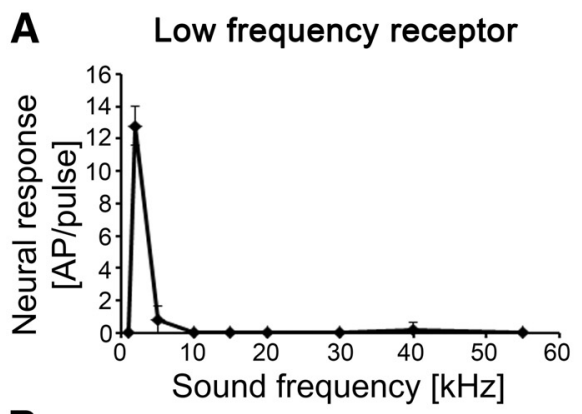

Broad-band frequency receptor

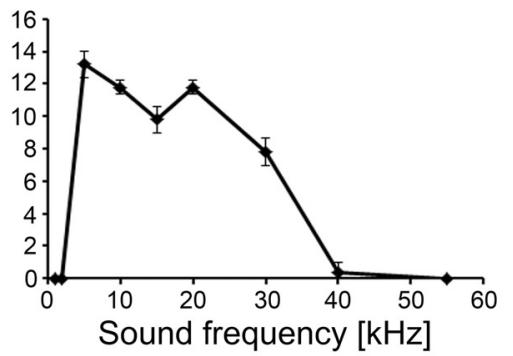

B

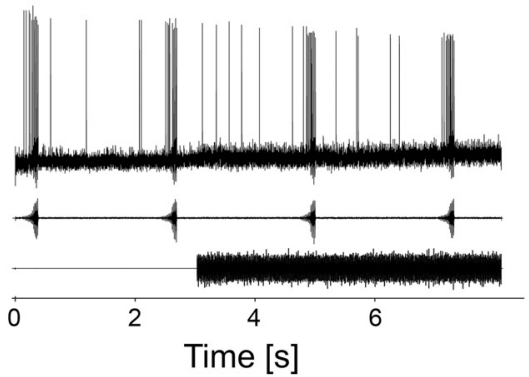

C
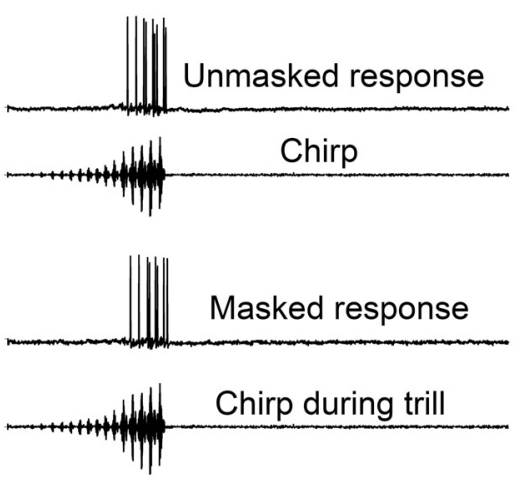

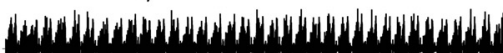

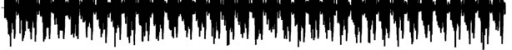
$\begin{array}{cccc}0 & 200 & 400 \quad 600 & 800 \\ & \text { Time [s] }\end{array}$
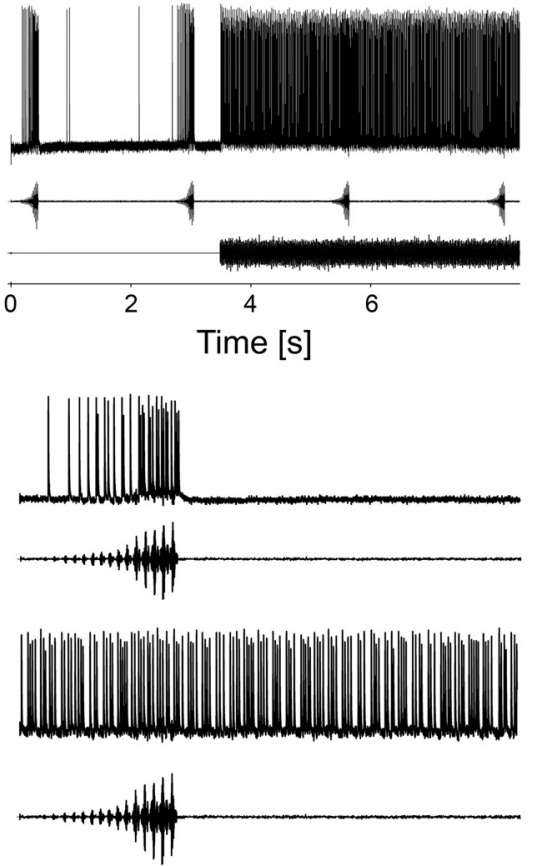

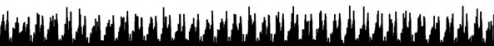

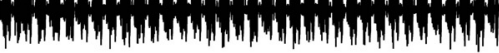

$\begin{array}{lllll}0 & 200 & 400 & 600 & 800\end{array}$

Time $[\mathrm{s}]$

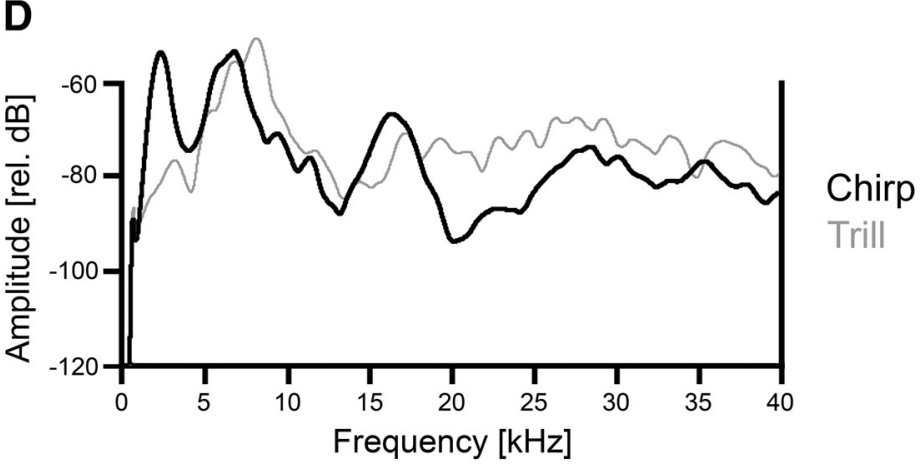

Figure 1. Frequency tuning and responses of a low-frequency and broadband frequency receptor to a chirp with and without the masking trill. $\boldsymbol{A}$, Iso-intensity response functions at $80 \mathrm{~dB}$ SPL. Error bars show standard deviation (SD) values. $\boldsymbol{B}$, Recording traces of receptors when the chirp was presented alone and under the masking trill. $C$, Responses of the same receptors to a chirp without trill (top) and a masked chirp (bottom) at sound intensities of $75 \mathrm{dBSPL}$. D, Spectrograms of the chirp (black, thick line) and trill (gray, thin line) recorded at the position of the preparation. Note the higher amplitude at $2 \mathrm{kHz}$ in the chirp spectrum.

\section{Results}

Response selectivity of auditory receptors

Approximately 40 sound receptors are arranged in a linear fashion in the crista acustica in the ears of the forelegs (Strauß et al., 2012). They are individually tuned to different frequencies from 
A
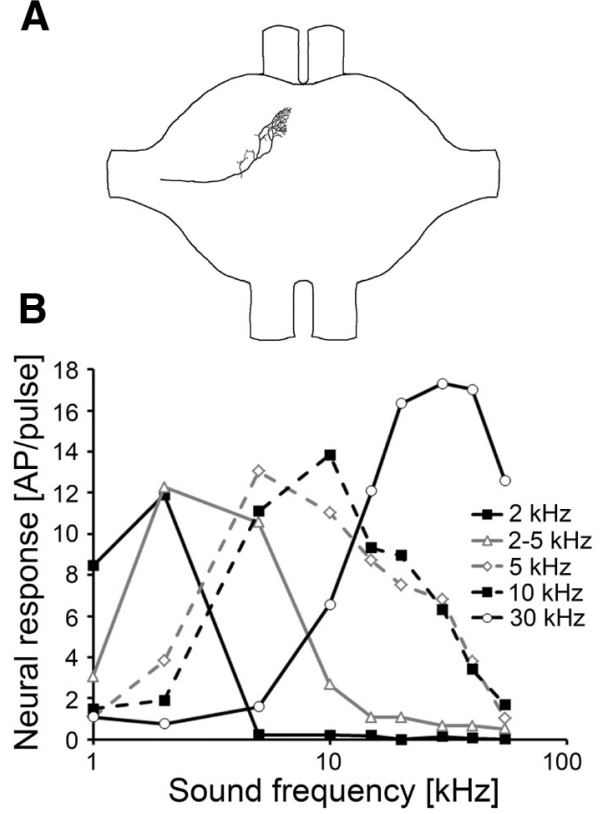

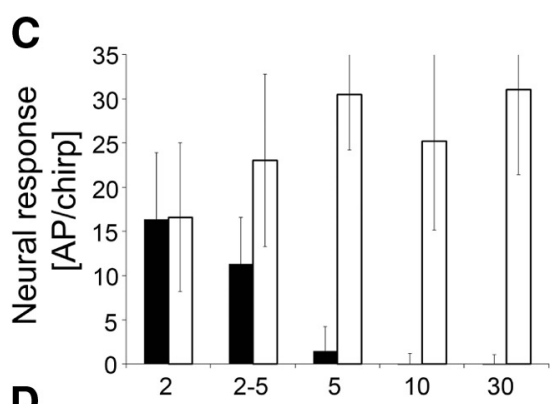

D

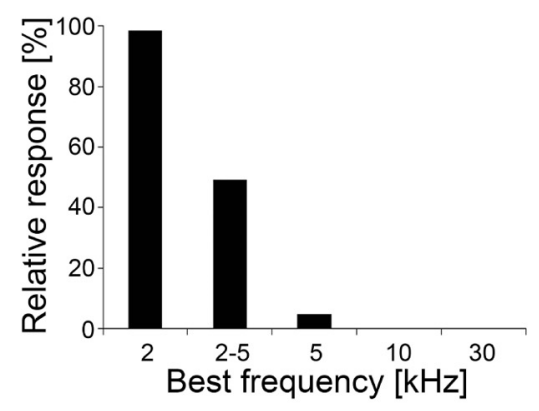

Figure 2. Axonal branching pattern, frequency tuning, and response selectivity for conspecific chirps of different auditory receptors. $\boldsymbol{A}$, Axonal branching pattern of a low-frequency receptor within the auditory neuropil of the prothoracic ganglion. $\boldsymbol{B}$, Iso-intensity function of different receptors at $80 \mathrm{~dB} \mathrm{SPL}(N=9$ for $2 \mathrm{kHz}, N=9$ for $2-5 \mathrm{kHz}, N=6$ for $5 \mathrm{kHz}, N=9$ for $10 \mathrm{kHz}$, and $N=7$ for $30 \mathrm{kHz}$ receptors). C, Average responses to the chirp presented alone (open bars) and under the masking trill (black bars) for receptors tuned to $2,2-5,5,10$, and $30 \mathrm{kHz}$ (mean \pm SD; $N$ as in $\boldsymbol{B}$ ). $\boldsymbol{D}$, Relative values of response magnitude to masked chirps (100\% = magnitude of responses to unmasked chirps).

$\sim 2$ to $60 \mathrm{kHz}$, similar to other katydids (Römer, 1983; Kalmring et al., 1990; Stumpner, 1996; Stolting and Stumpner, 1998). To understand the neural mechanisms underlying signal detection and recognition under masking conditions, we first analyzed the tuning and neural responses to chirps and trills in various auditory receptors. Figure $1 A$ shows the tuning of two auditory receptors as iso-intensity response profiles. The low-frequency receptor (LFR) responded highly selective to $2 \mathrm{kHz}$, with almost no response to lower or higher frequencies, even though sound pulses were broadcast at $80 \mathrm{~dB}$ SPL. In contrast, the broadband frequency receptor (BFR) generated a strong response from 5 to $30 \mathrm{kHz}$. The spectral composition of the chirp and trill and the consequences of the different tuning of receptors for their selectivity are shown in Figure 1. The LFR responded only to the chirp; even when both chirp and trill were presented simultaneously at $75 \mathrm{~dB}$ SPL its response was completely unaffected by the trill (Fig. $1 C)$. In contrast, the BFR showed a strong, continuous response to the trill that completely masked the responses to the chirp, leading to a dramatic decrease of the neural representation of the chirp under masking (Fig. 1C). The quantitative data for the responses to the chirps and the chirps masked by the trill are 17.7 and 16.1 action potentials (APs), respectively, for the LFR and 24.4 and $1 \mathrm{APs}$, respectively, for the BFR. Thus, there is a strong reduction of response under masking conditions for the latter. The axonal arborizations of the LFR receptor are in the most anterior areas of the auditory neuropil (Fig. 2A) similar to those reported previously in other katydids (Römer, 1983; Stumpner, 1996; Stolting and Stumpner, 1998) and thus represent auditory receptors from the crista acustica and not the intermediate organ. Figure $2 B$ shows iso-intensity response functions of different receptors. The LFRs tuned to $2 \mathrm{kHz}$ generated on average $11.9 \pm 3.8$ APs per stimulus at the best frequency, while the high-frequency receptors (HFRs) tuned to $30-40 \mathrm{kHz}$ responded with $17.3 \pm 4.3$
APs per stimulus at the best frequency. These differences are statistically significant $(p=0.034$; Mann-Whitney rank sum test). Additionally, the iso-intensity response functions of LFRs tend to be more selective than those of HFRs. Receptors tuned to $2 \mathrm{kHz}$ revealed a high sensitivity only within the narrow frequency range of $1-2 \mathrm{kHz}$, while high-frequency afferents tuned to $30-40 \mathrm{kHz}$ showed suprathreshold responses from 15 to $55 \mathrm{kHz}$ (and probably higher). This difference can be partly explained by the higher absolute sensitivity of HFRs; tuning curves based on hearing thresholds would probably reveal more similar frequency bandwidths in LFRs and HFRs as shown for other katydids (Römer, 1983; Stumpner, 1996; Stolting and Stumpner, 1998).

Figure $2 C$ shows averaged responses of differently tuned receptors to chirps with and without the masking trill. The magnitude of responses to chirps without the masker is generally stronger in receptors tuned to frequencies higher than $2 \mathrm{kHz}$ $(31.0 \pm 9.6 \mathrm{APs}$ per chirp in $30 \mathrm{kHz}$ receptors as compared with16.6 \pm 8.4 APs per chirp in $2 \mathrm{kHz}$ receptors at $75 \mathrm{~dB}$ SPL). However, in the presence of the trill the responses to the chirp are unaffected in receptors tuned to $2 \mathrm{kHz}$, but decrease to only $1.5 \pm 2.8$ APs per chirp in $5 \mathrm{kHz}$ receptors and nearly zero in 10 and $30 \mathrm{kHz}$ receptors.

Figure $2 D$ illustrates this finding using the relative values of the responses to the chirp under both conditions. The sharply tuned $2 \mathrm{kHz}$ receptor was unaffected by the higher frequencies of the trill and revealed an activity of $100 \%$ when stimulated with chirps under the masker. The more broadly tuned $2 \mathrm{kHz}$ LFR, however, showed a significant reduction by $50 \%$ and the $5 \mathrm{kHz}$ receptor responded with only $4.8 \%$ to the chirp under noise. Receptors tuned to $10 \mathrm{kHz}$ or $30 \mathrm{kHz}$ revealed no chirp-specific response at all when the chirp was broadcast simultaneously with the trill.

\section{Two mechanisms in interneurons for signal detection under masking trill conditions}

Based on the data of auditory receptors, we can conclude that sharply tuned LFRs respond selectively to the $2 \mathrm{kHz}$ component of the chirp, a characteristic that can be exploited at higher levels of auditory processing.

\section{Selective tuning}

Similar to the sharply tuned LFRs, some interneurons exhibit response selectivity for the chirp under masking conditions by being selectively tuned to the low-frequency component of the chirp at $2 \mathrm{kHz}$. We identified three auditory interneurons that revealed a tuning similar to sharply tuned LFRs. Their EPSP delay of 1-2 ms relative to that of auditory afferents, and the overlap of dendritic arborizations with axonal projections of LFRs (Fig. 2 A), indicate that they receive direct input from auditory receptors.

Figure $3 A$ shows reconstructions of three Alexa 555-labeled interneurons tuned to low frequencies. LFT- 1 has bilateral symmetrical dendritic arborizations in the auditory neuropil, a T-shaped 

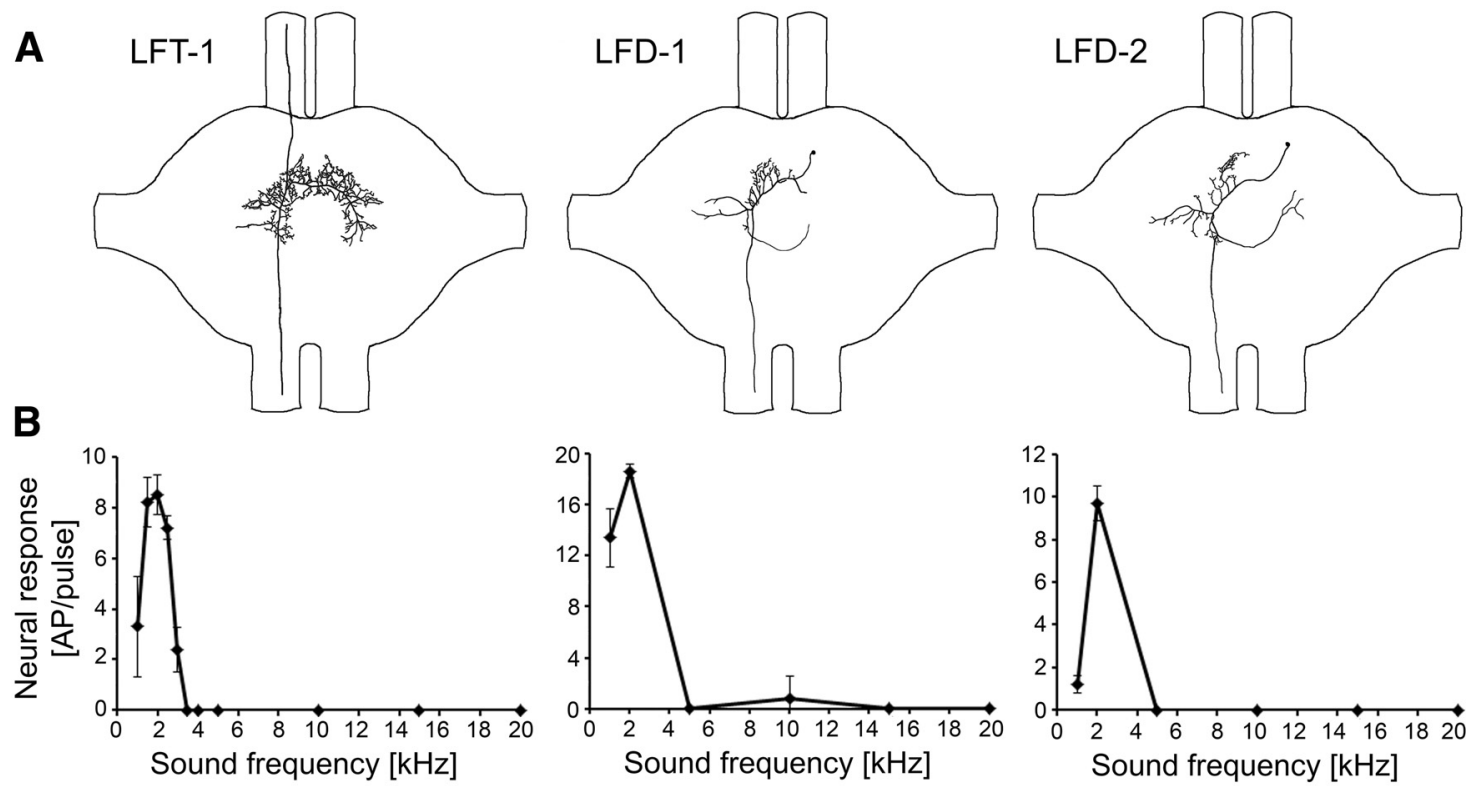

C
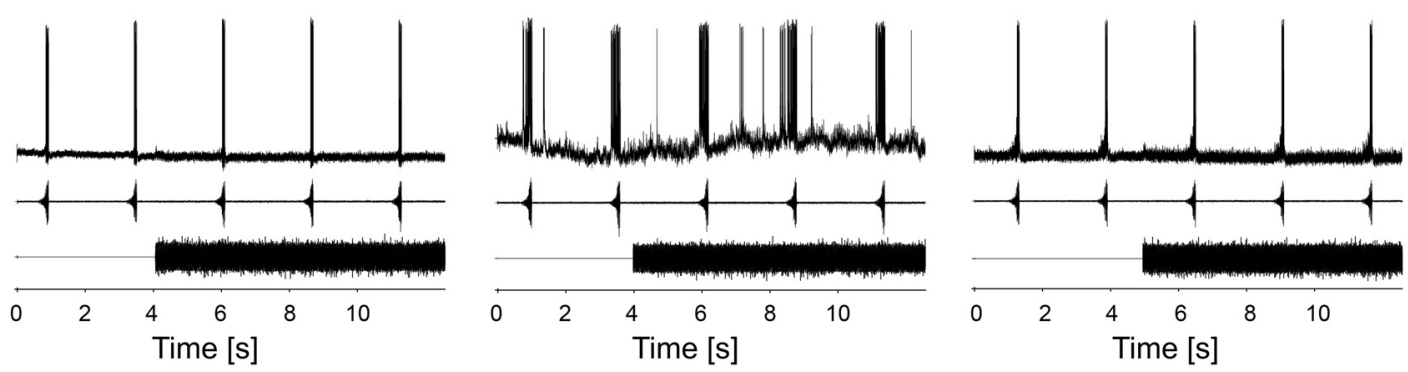

D
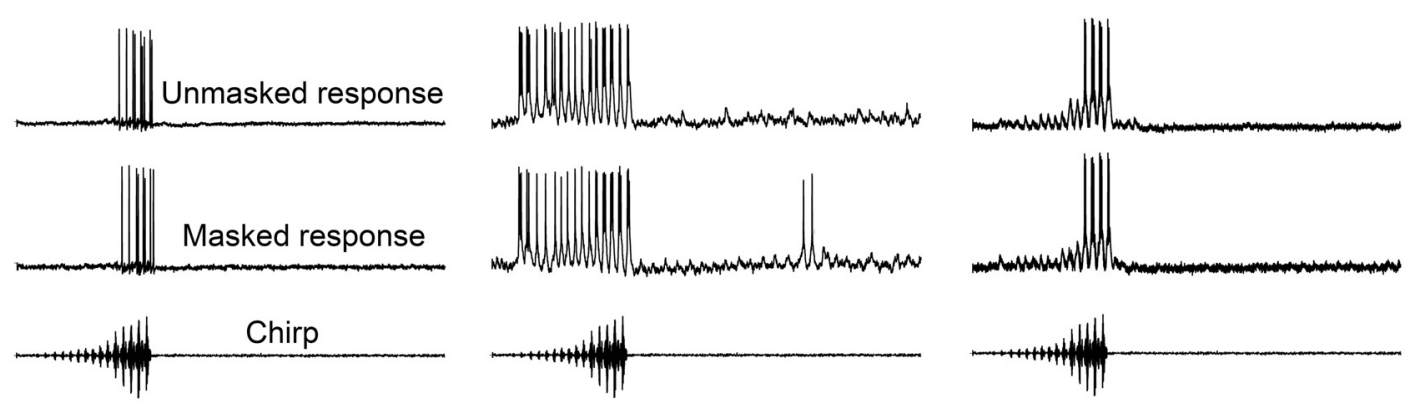

Figure 3. Structure, frequency tuning, and response selectivity to chirps of LFIs. $A$, Reconstructions of intracellular stainings of three types of LFls in the prothoracic ganglion. $\boldsymbol{B}$, Iso-intensity response functions of LFIs at $80 \mathrm{~dB}$ SPL. Error bars show SD values. C, Characteristic examples of LFI responses to chirps presented alone and under the masking trill. $\boldsymbol{D}$, Characteristic examples of responses of LFIs to a chirp without trill (top) and a masked chirp (bottom).

structure with an ascending axon toward the subesophageal ganglion and a descending axon toward the mesothoracic ganglion. Its cell body is not located within the prothoracic ganglion. Two other interneurons (LFD-1 and LFD-2) have axons descending to the mesothoracic ganglion; they show similar branching patterns, but differed considerably in threshold and response magnitude. We therefore decided to separate these neurons, mainly because the difference in the excitation level was very constant, with no intermediate level, which one would expect if there were some unknown reasons modifying the sensitivity between preparations. Their cell body is located near the anterior auditory neuropil; the dendrites branched contralateral to the cell body within the anterior part of the auditory neuropil. Typical features were also dendritic branches toward the leg nerve and a semicircular projection to the ipsilateral side of the cell body within the posterior part of the neuropil.

The three interneurons were all sharply tuned to $2 \mathrm{kHz}$ (Fig. $3 B$ ); almost no response occurred at $3.0 \mathrm{kHz}$ and higher. They differed, however, in sensitivity, so that at $75 \mathrm{~dB}$ SPL LFD-1 showed a strong response even for the low-amplitude syllables of the chirp (approximately $-20 \mathrm{~dB}$ ), whereas LFT-1 and LFD-2 responded reliably only to the last syllables. Similar to the $2 \mathrm{kHz}$ receptors, the response of all three neurons to the chirp was unaffected by the masking trill (Fig. $3 C, D$ ). Note that LFD-1 showed even a selective response to the chirp at an SNR of $-21 \mathrm{~dB}$ (the trill being $21 \mathrm{~dB}$ more intense than the chirp). Quantitative average values for all three neurons and their responses with and without the masking trill are given in 
A

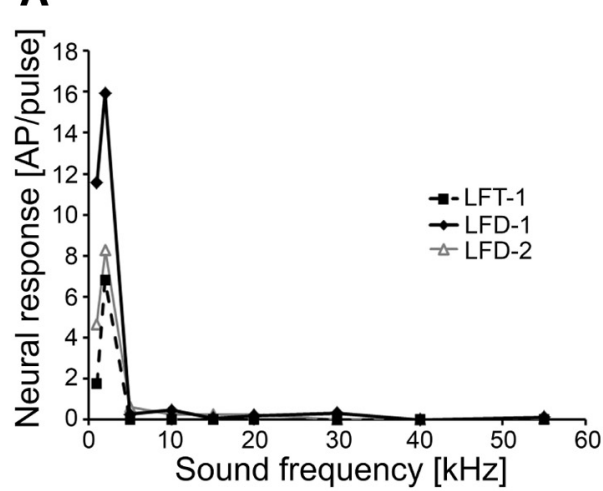

B

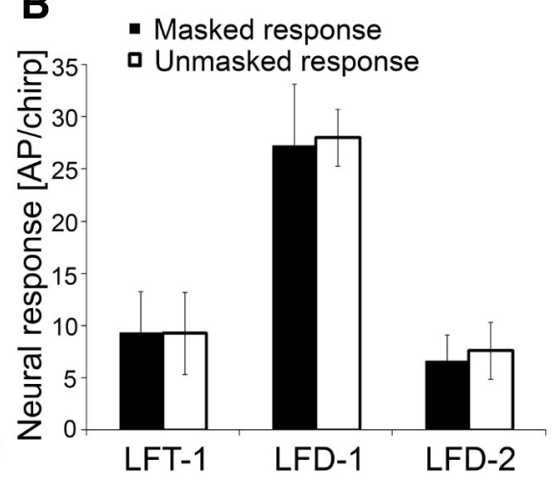

Figure 4. A, Comparison of iso-intensity response functions of LFIs at $80 \mathrm{~dB} S P L$ ( $N=3$ for LFT-1, $N=3$ for LFD-1, and $N=4$ for LFD-2). $B$, Average responses of LFls to chirps in the unmasked and masked condition (white and black bars, respectively; $N=$ 4 for LFT-1, $N=4$ for LFD-1, and $N=5$ for LFD-2). Error bars show SD values.

Figure 4. LFT-1 and LFD-2 responded with 9.2 and 8.1 APs to the unmasked chirp, respectively, and the more sensitive LFD-1 neuron generated 28 APs per chirp. There was no significant difference in the responses under masked and unmasked conditions.

\section{Novelty detection}

A second mechanism for selective responses to chirps exists in interneurons that are tuned to a broader range of frequencies. We identified three interneurons where the response to the continuous trill exhibits strong adaptation. In contrast, the response to the chirp is unaffected by the adaptation because of the $2 \mathrm{kHz}$ component of the chirp not present in the trill (novelty detection; as suggested by Schul and Sheridan, 2006 and Schul et al., 2012).

The structures of these three interneurons in the prothoracic ganglion are shown in Figure 5A. The cell body of BFD-1 is located near the anterior border of the auditory neuropil and has a contralateral axon descending to the mesothoracic ganglion. Although its structure is similar to LFD-1 and LFD-2, its dendrites on both sides of the ganglion reveal consistent differences. BFA-1 has its cell body close to the dorsal surface of the anterior lobes and dendrites contralateral to the cell body and projects with an ascending axon to the subesophageal ganglion. BFT-1 has a descending and ascending axon and dendrites within the auditory neuropil. Its cell body is not located within the prothoracic ganglion.

These neurons respond to a broad range of frequencies, as evident in their iso-intensity function (Fig. 5B). A characteristic feature of all BFD-1 neurons is a peak of activity at $2 \mathrm{kHz}$, in addition to a broad frequency range at higher sonic and ultrasonic frequencies. In contrast, BFA-1 and BFT-1 showed a broadband tuning with strongest responses toward higher frequencies, but also including sensitivity to $2 \mathrm{kHz}$.

A common property of these interneurons is their strong adaptation to the onset of the trill (Fig. 5C). The time course of adaptation varies strongly between these neurons from several seconds in BFD-1 and BFA- 1 to $<100 \mathrm{~ms}$ in BFT-1. As a consequence, although the response to the chirp may be completely masked at the onset of the masking trill, it recovers due to adaptation. Different to the interneurons tuned exclusively to the 2 $\mathrm{kHz}$ component of the chirp, these responses under the masking trill after some seconds of adaptation are weaker compared with the unmasked condition (Fig. 5C,D). The comparison of response strength between the unmasked and masked condition shows differences between the three interneurons (8.3 and 4.4
APs per chirp in BDF-1, 34.8 and 15.4 APs per chirp in BFA-1, and 11.3 and 4.3 APs per chirp in BFT-1). However, even at an SNR of $-15 \mathrm{~dB}$, a chirp elicited a burst of 10 APs in BFA-1.

A neural mechanism for a stimulus selectivity based on novelty detection requires that only a specific stimulus component (like the $2 \mathrm{kHz}$ component in the chirp) will elicit a response despite strong adaptations to other components in the continuous background. We tested this hypothesis by reducing the amplitude of the $2 \mathrm{kHz}$ component in the chirp to the same level as in the trill (Siegert et al., 2013). Figure $6 A$ shows responses of BFD-1 to chirps with and without the 2 $\mathrm{kHz}$ frequency component. Unmasked chirps with the reduced $2 \mathrm{kHz}$ component elicited a strong response in this broadband neuron because of the high frequencies in the chirp, but under the masking condition of the trill there was no response to the chirp when the $2 \mathrm{kHz}$ component was reduced (Fig. $6 \mathrm{~A}$, right).

The averaged results from such experiments in three BFD-1 neurons are shown in Figure 6B. In the masking condition of the trill, chirps elicited a reliable burst of APs, although reduced compared with the unmasked condition $(7.3 \pm 3.9$ APs per chirp compared with $20.8 \pm 10.3$ APs per chirp). However, when the 2 $\mathrm{kHz}$ component in the chirp was equalized to the spectrum of the trill, the masked response in BFD- 1 was almost absent $(0.6 \pm 0.6$ APs per chirp compared with $17.3 \pm 3.2$ APs per chirp). These data reveal that the difference in frequency composition at $2 \mathrm{kHz}$ between the conspecific signal and the heterospecific masker is essential for the mechanism of novelty detection. An important physiological characteristic in neurons that use a novelty detection mechanism is that the response to the signal is reduced due to adaptation to the continuous masker, but the masker evokes subthreshold EPSPs (see EPSPs in bottom of Fig. 6A). These EPSPs match precisely with the double syllables of the trill (Fig. 6C).

Quantitative values for all broadband neurons regarding tuning properties and chirp selectivity under noise are given in Figure 7. Similar to the representative neurons of Figure 5, the averages over more neurons revealed a bimodal activity for BFD-1 and a broadband tuning for BFA-1 and BFT-1 (Fig. 7A). These tuning properties have a significant impact on chirp selectivity, both in the unmasked and masked condition (Fig. 7B). Although BFT-1 revealed the lowest sensitivity it also showed the strongest adaptation to the trill leading to a better neural representation of the chirp under noise.

Table 1 emphasizes the degree of chirp selectivity for all identified interneurons by comparing responses to noise with responses to chirps under noise. The table includes all neurons that use either the mechanisms of frequency selectivity or novelty detection and revealed a statistically significant difference between the responses to the masking trill and the responses to the masked chirp.

As mentioned before, the selective tuning to the $2 \mathrm{kHz}$ component in the chirp and the novelty detection mechanism provide selective responses in interneurons to the chirp at remarkably low SNRs. In LFD-1 and BFA-1 we found two different dependencies of masked responses when systematically varying the SNR from 0 $\mathrm{dB}$ to the masked threshold (Fig. 8). The masked response in LFD- 1 at $0 \mathrm{~dB}$ SNR is rather strong with $28.8 \pm 2.6$ APs per chirp, 

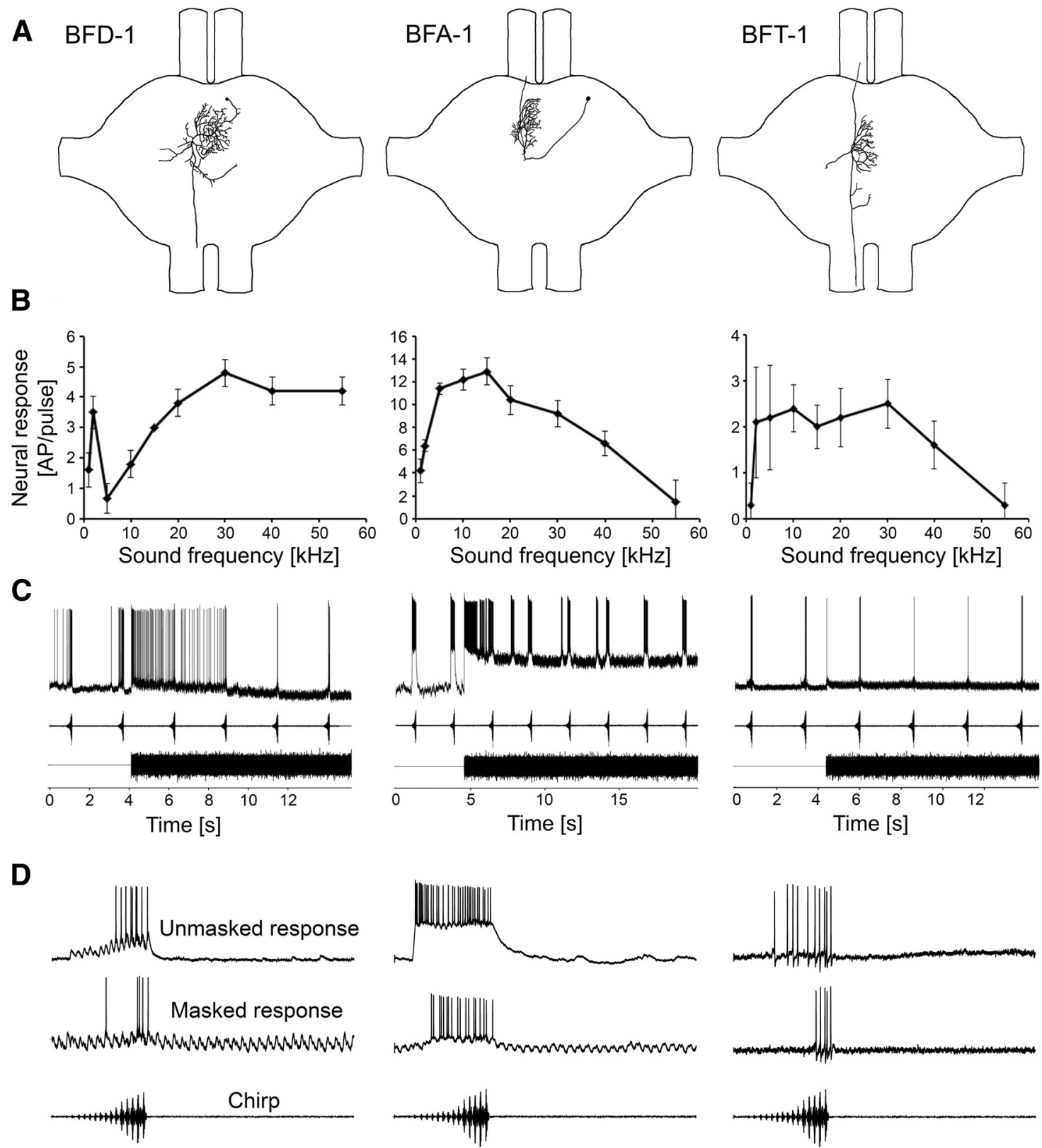

Figure 5. Structure, frequency tuning, and response selectivity to chirps of three types of broadband interneurons. $\boldsymbol{A}$, Reconstructions of intracellular stainings in the prothoracic ganglion. $\boldsymbol{B}$, Iso-intensity response functions at $80 \mathrm{~dB}$ SPL. Error bars show SD values. C, Characteristic examples of responses to chirps presented alone and under the masking trill. $\boldsymbol{D}$, Characteristic examples of responses to a chirp without trill (top) and a masked chirp (bottom).

and it gradually declines with decreasing SNRs, so that there is still a reduced, but reliable, response of $3.9 \pm 3.0$ APs per chirp at $-18 \mathrm{~dB}$ SNR (Fig. $8 B$ ). In contrast, the masked responses in BFA-1 vary much less with decreasing SNR values, but the response at $-18 \mathrm{~dB}$ SNR is still reliable with $3.0 \pm 0.8$ APs per chirp. To get an impression of the afferent information available for the insect at a reduced SNR of $-15 \mathrm{~dB}$ via all identified interneurons, we show their representative responses in Figure $8 C$.

\section{Discussion}

The acoustic environment of many species with vocal communication can be characterized by what is commonly known as the cocktail-party problem, where receivers have to extract acoustic objects from a mixture of sound waves arriving at the ears from various sound sources in space. We have chosen to study solutions for a cocktail party-like problem in an insect, where signal detection is extremely difficult, because the signal (the chirp) shares most of the frequency spectrum with the long-lasting, intense masking trill of a competing species in the same habitat, in addition to the general high acoustic background in the nocturnal rainforest (Lang et al., 2005). We would expect to find robust solutions to the masking problem in the afferent processing of sensory information in insects with their limited number of neurons available (Schmidt and Römer, 2011; Hildebrandt et al., 2015). Such processing would free the CNS from the complicated task of distinguishing between afferent activity resulting from background noise and relevant signals, which is also the core of the matched-filter hypothesis (Capranica and Moffat, 1983; Wehner, 1989).

The solutions we describe are based on a small difference in the spectral composition between the chirp and the trill at $2 \mathrm{kHz}$, a component that is more intense in the chirp (Fig. 1D). The two 
A $+2 \mathrm{kHz}$ component _.nummum Unmasked response W I

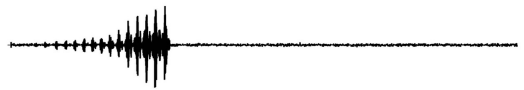

B

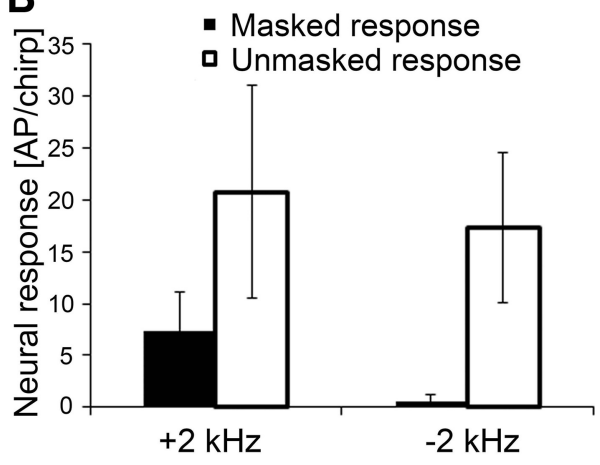

C Average of EPSPs
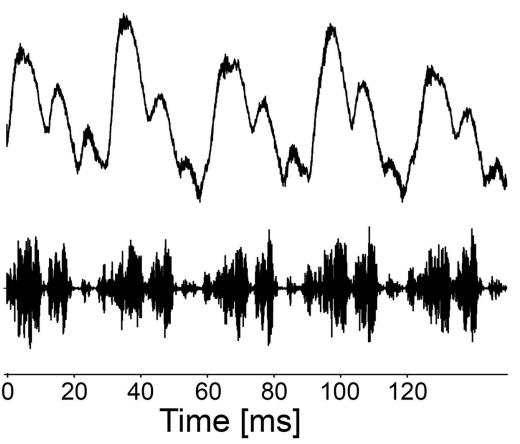

Figure 6. Importance of the $2 \mathrm{kHz}$ component in the chirp for release from masking. $\boldsymbol{A}$, Characteristic intracellular responses of BFD-1 to chirps including the $2 \mathrm{kHz}$ component (left) and to chirps with the $2 \mathrm{kHz}$ component reduced through high-pass filtering (right). Responses are given for unmasked and masked condition (top and bottom recording, respectively). Note the ongoing, subthreshold EPSP activity in response to the continuous trill. $\boldsymbol{B}$, Average responses to chirps with and without the $2 \mathrm{kHz}$ component ( $N=3 \pm$ SD BFD-1 neurons). C, Averaged EPSPs in a BFD-1 neuron in response to the double syllables of the trill.
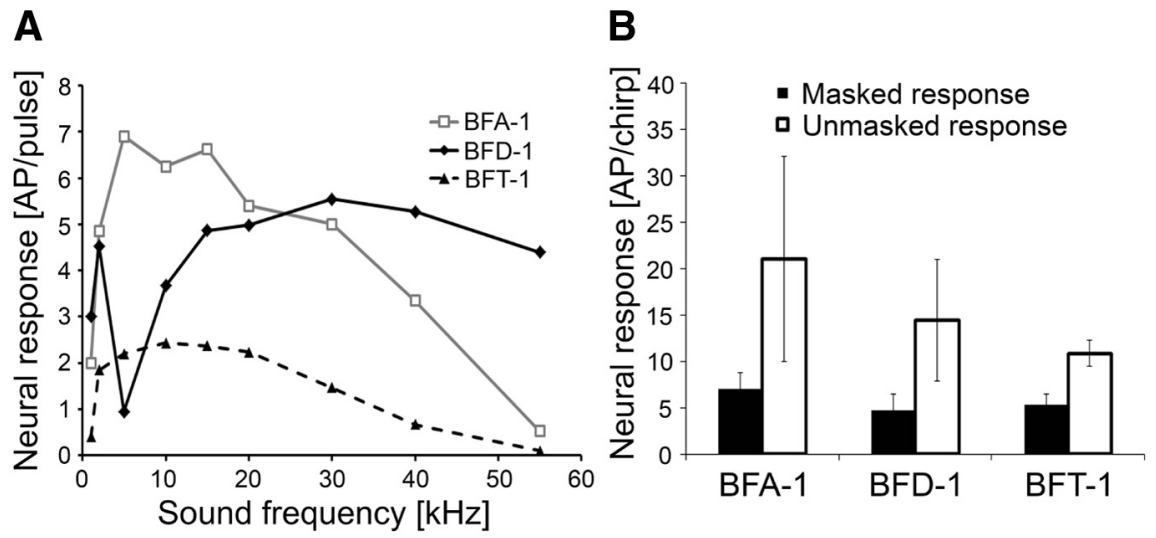

Figure 7. A, Comparison of iso-intensity functions of broadband frequency interneurons (BFIs) at $80 \mathrm{~dB} \mathrm{SPL}$ ( $N=4$ for BFA-1, $N=9$ for BFD-1, and $N=3$ for BFT-1). $B$, Average responses of BFls to chirps in the unmasked and masked condition (white and black bars, respectively; $N=5$ for BFA-1, $N=9$ for BFD-1, and $N=3$ for BFT-1). Error bars show SD values.

Table 1. Test for significant differences between responses to the noise alone compared with responses to the chirp under noise (paired $t$ test)

\begin{tabular}{lllllll}
\hline Interneurons & LFT-1 & LFD-1 & LFD-2 & BFD-1 & BFA-1 & BFT-1 \\
\hline Individuals & $N=4$ & $N=4$ & $N=5$ & $N=9$ & $N=5$ & $N=3$ \\
$p$ value & $p=0.017$ & $p=0.005$ & $p=0.004$ & $p<0.001$ & $p<0.001$ & $p=0.014$ \\
\hline
\end{tabular}

All neurons using selective tuning or novelty detection revealed statistically significant differences $(p<0.05)$.

neural mechanisms of selective tuning and novelty detection are both based on this small difference between the signal and the masker. They may represent the neuronal basis for the behavioral ability of males to detect and synchronize their calling songs even under the masking trills of the competing species (Siegert et al.,
2013). At the receptor level, some receptors responded selectively to chirps masked by the trill due to their narrow tuning for $2 \mathrm{kHz}$. These responses were almost identical when chirps were presented with or without the masker, as high-frequency components of the trill failed to elicit any response. In contrast, receptors sensitive at higher frequencies generated strong responses to the trill, and the neural representation of the chirp was completely masked by the activity to the trill. This sheds new light on the ability of katydids for peripheral frequency discrimination, where recent studies have demonstrated the sophisticated biophysical mechanisms underlying the tonotopic representation of frequencies in their ear (Palghat Udayashankar et al., 2012; Montealegre-Z et al., 2012), but most of the detailed frequency representation appears to be lost in afferent interneurons due to strong neuronal convergence from receptors onto interneurons. This convergence would completely abolish any detailed frequency information, so that differences in the frequency content of two signals, or signal and masker, could not be used any more for discrimination.

However, our present findings clearly indicate that selective tuning to a particular component of an otherwise broad frequency range in a conspecific signal may provide the basis for successful listening under strong masking conditions. At the level of auditory interneurons we identified two neural mechanisms underlying masked signal detection. The first is based on selective tuning to the low-frequency components of the chirp. A similar mechanism was described by Schul (1999) for song discrimination in Tettigonia viridissima. Consistent with the tonotopy of the auditory neuropil (Römer, 1983; Stolting and Stumpner, 1998), the dendrites of these neurons overlap with the axonal arborizations of the low-frequency receptors. Apparently, these neurons receive only inputs from sharply tuned lowfrequency receptors, and inhibitory mechanisms to sharpen the tuning of a neuron for selective coding of relevant stimuli are not necessary (Stumpner, 1998).

The second mechanism for signal detection under noise is based on novelty detection as described previously (Schul and Sheridan, 2006; Schul et al., 2012; Siegert et al., 2013). The mechanism has two characteristics: broadband interneurons are excited with bursts of APs at the onset of the trill, but show subsequent strong adaptation. In the adapted state they respond only to the frequency component not present in the trill, i.e., the $2 \mathrm{kHz}$ "novelty" of the chirp. Thus, when the $2 \mathrm{kHz}$ component was equalized to the level of the trill, all components of the chirp were completely masked by the trill and no "novel" signal trait 
could elicit a response (Fig. 6). However, these interneurons exploit the entire frequency range for responses to unmasked chirps.

Schul and Sheridan (2006) described a similar mechanism for the detection of a bat echolocation signal in the presence of a continuous call of a conspecific with reliable responses to the bat call at SNRs of $-18 \mathrm{~dB}$ at the masked threshold. Triblehorn and Schul (2013) suggested a model that stimulus-specific adaptation may occur in the dendrites for one signal without preventing a response to the other signal. Prerequisites for this model are tonotopic projections of auditory receptors within the auditory neuropil and their overlap with dendritic branches of interneurons (Römer, 1983; Römer et al., 1988; Ebendt et al., 1994). Such a model would fit quite well with our current results, since the interneurons receive low-frequency input at dendritic areas that are separated from those to high-frequency inputs, allowing them to generate suprathreshold responses during adaptation.

Our data indicate that LFRs are less sensitive compared with HFRs as the excitation at best frequency is lower (Fig. 2). As the sensitivity for the $2 \mathrm{kHz}$ component is crucial for the response selectivity to chirps (Fig. 6), we would expect a breakdown of selectivity when the $2 \mathrm{kHz}$ component falls below threshold at higher sender-receiver distances. However, in the natural habitat low frequencies suffer much less from excess attenuation compared with higher frequencies (Romer and Lewald, 1992). It is thus likely that at higher communication distances the $2 \mathrm{kHz}$ component can still activate LFRs, due to the low-frequency advantage in sound transmission, although their absolute sensitivity is reduced compared with HFRs.

Apparently, both mechanisms of selective low-frequency tuning and novelty detection are highly adaptive under the specific ecological conditions of acoustic communication for the Mecopoda chirper, because they provide reliable representation of the conspecific signal at unfavorable SNRs (Fig. 8). A comparison with homologous neurons in other katydid and cricket species may possibly provide information whether and how existing properties of auditory receptors and interneurons in Mecopoda have evolved as a result of the ecological constraints of strong masking.

For example, interneurons with similar morphology as LFD-1 or other LFD-interneurons have been described in other katydids and crickets, respectively (Wohlers and Huber, 1982; Römer, 1987). The DN1-neuron in crickets, for instance, is tuned to low frequencies between 1 and $3 \mathrm{kHz}$, but with a broader tuning as compared with LFD neurons. It is thus possible that the Mecopoda chirper recruited already existing interneurons serving other function in other species - by sharply tuning the input to 2 $\mathrm{kHz}$ the representation of masked chirps is greatly enhanced. BFA-1 interneurons may represent a similar case. They may be homologous to ascending neurons AN1-AN3 described by Stumpner (1997) and Stumpner and Molina (2006) in Ancistrura nigrovittata; however, these neurons were tuned to higher frequencies compared with BFA-1 in Mecopoda. Again, these tuning properto $2 \mathrm{kHz}$, and for BFA-1 and BFD-1.
LFD-1

BFA-1

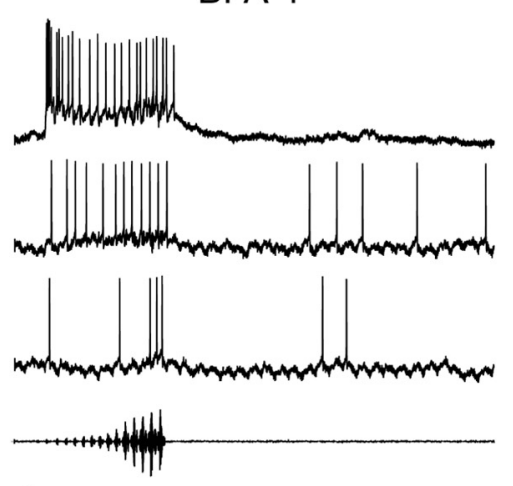

C

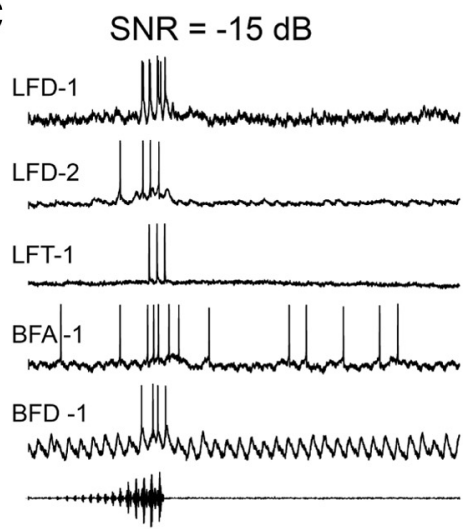

Figure 8. Responses of LFD-1 and BFA-1 to chirps at different SNRs. $A$, Representative responses in the unmasked and masked show SD values. $C$, Representative traces of responses to the masked chirp at an SNR of $-15 \mathrm{~dB}$ for the three interneurons tuned

ties of broadband interneurons may easily result from modifications of synaptic input from auditory receptors to the dendrites over evolutionary time for the specific task to detect the $2 \mathrm{kHz}$ component in the masked signal. One of the most obvious cases for a small, but functionally important evolutionary modification is BFD-1 with its second peak of sensitivity sharply restricted at 2 $\mathrm{kHz}$. This low-frequency peak in sensitivity would strongly suggest that it is an evolutionary innovation providing one of the two requirements for novelty detection: given that the dendritic mechanisms contributing to stimulus-specific adaptation had already been established (Triblehorn and Schul, 2013), the addition of a sharply tuned $2 \mathrm{kHz}$ input to an existing broad frequency would make such a neuron an ideal candidate for detecting the chirp under the masking trill of the competing species.

In this context, an obvious series of experiments would require a comparison of homologous interneurons in the two competing species, the Mecopoda chirper and the trilling species, since such comparison may provide significant insights into how neural mechanisms of signal detection under noise are shaped by evolution.

An important further goal is to reveal the projection areas of the described ascending and descending interneurons and link them to pattern recognizing and/or motor control areas. The projections of descending interneurons may be linked to motoneurons of flight muscles and/or motor control areas for phonotaxis in females. Accordingly, the two mechanisms may be used for different tasks depending on their projection areas. Interneurons that exploit a broader frequency range and generate responses with robust representations of the temporal signal pattern over distance (BFA-1) may be used for pattern recogni- 
tion, while the very fast response to the onset of chirps of lowfrequency interneurons (LFIs) may be involved in more direct pathways for motor control (LFD-1). A parallel, faster and more direct pathway for motor control has been proposed for crickets (Hedwig and Poulet, 2004, 2005), which is activated once the conspecific pattern has been recognized.

\section{References}

Bee MA, Micheyl C (2008) The cocktail party problem: what is it? How can it be solved? And why should animal behaviorists study it? J Comp Psychol 122:235-251. CrossRef Medline

Bradbury JW, Vehrenkamp SL (2011) Principles of animal communication. Sunderland, MA: Sinauer.

Bregman AS (1990) Auditory scene analysis: the perceptual organization of sound. Cambridge, MA: MIT.

Bronkhorst AW (2000) The cocktail party phenomenon: a review of research on speech intelligibility in multiple-talker conditions. Acta Acustica $86: 117-128$.

Brumm H, ed (2013) Animal communication and noise, animal signals and communication, Vol 2. New York: Springer.

Brumm H, Slabbekoorn H (2005) Acoustic communication in noise. In: Advances in the study of behavior, Vol 35, pp 151-209. San Diego, CA: Academic.

Capranica RR, Moffat AJM (1983) Neurobehavioral correlates of sound communication in anurans. In: Advances in vertebrate neuroethology (Ewert JP, Capranica RR, Ingle D, eds), pp 701-730. New York: Plenum.

Cherry EC (1953) Some experiments on the recognition of speech with one and with two ears. J Acoust Soc Am 25:975-979. CrossRef

Ebendt R, Friedel J, Kalmring K (1994) Central projection of auditory receptors in the prothoracic ganglion of the bushcricket Psorodonotus illyricus (Tettigoniidae): computer-aided analysis of the end branch pattern. J Neurobiol 25:35-49. CrossRef Medline

Greenfield MD (1988) Interspecific acoustic interactions among katydids Neoconocephalus: inhibition-induced shifts in diel periodicity. Anim Behav 36:684-695. CrossRef

Hedwig B, Poulet JF (2004) Complex auditory behavior emerges from simple reactive steering. Nature 430:781-785. CrossRef Medline

Hedwig B, Poulet JF (2005) Mechanisms underlying phonotactic steering in the cricket Gryllus bimaculatus revealed with a fast trackball system. J Exp Biol 208:915-927. CrossRef Medline

Higham JP, Hebets EA (2013) An introduction to multimodal communication. Behav Ecol Sociobiol 67:1381-1388. CrossRef

Hildebrandt KJ, Benda J, Hennig RM (2015) Computational themes of peripheral processing in the auditory pathway of insects. J Comp Physiol A Neuroethol Sens Neural Behav Physiol 201:39-50. CrossRef Medline

Hulse SH (2002) Auditory scene analysis in animal communication. Adv Study Behav 31:163-200.

Kalmring K, Ebendt R, Ahi J, Hellweg J, Young D, Halex H, Lakes R, Rössler W, Schröder J (1990) Comparative investigation on the morphology and physiology of the auditory receptor organs of seven species of bushcrickets. In: Sensory systems and communication in arthropods (Gribakin FG, Wiese K, Popov AV, eds) pp 41-247. Basel: Birkhäuser.

Keuper A, Kalmring K, Schatral A, Latimer W, Kaiser W (1986) Behavioural adaptations of ground living bushcrickets to the properties of sound propagation in low grassland. Oecologia 70:414-422. CrossRef

Knepper M, Hedwig B (1997) NEUROLAB, a PC-program for the processing of neurobiological data. Comput Methods Programs Biomed 52:7577. CrossRef Medline

Korsunovskaya OS (2008) Acoustic signals in katydids (Orthoptera, Tettigonidae). Communication I (Engl Transl). Entomol Rev 88:1032-1050. CrossRef

Krobath I (2013) Complex signaling, song interaction and mate choice in a trilling species of the Mecopoda complex (Orthoptera: Tettigoniidae). Master thesis, Karl-Franzens-University, Graz, Austria.

Lang A, Teppner I, Hartbauer M, Römer H (2005) Predation and noise in communication networks of neotropical katydids. In: Animal communication networks (McGregor P, ed.) pp152-169. Cambridge, UK: Cambridge UP.

Montealegre-Z F, Jonsson T, Robson-Brown KA, Postles M, Robert D (2012) Convergent evolution between insect and mammalian audition. Science 338:968-971. CrossRef Medline

Palghat Udayashankar A, Kössl M, Nowotny M (2012) Tonotopically ar- ranged traveling waves in the miniature hearing organ of bushcrickets. PLoS One 7:e31008. CrossRef Medline

Römer H (1983) Tonotopic organization of the bushcricket Tettigonia viridissima. Nature 306:60-62. CrossRef

Römer H (1987) Representation of auditory distance within a central neuropil of the bushcricket Mygalopsis marki. J Comp Physiol A Neuroethol Sens Neural Behav Physiol 161:33-42. CrossRef

Römer H (2013) Masking by noise in acoustic insects: problems and solutions. In: Animal communication and noise, animal signals and communication, Vol 2 (Brumm H, ed), pp 33-63.

Romer H, Lewald J (1992) High-frequency sound transmission in natural habitats: implications for the evolution of insect acoustic communication. Behav Ecol Sociobiol 29:437-444. CrossRef

Römer H, Marquart V, Hardt M (1988) Organization of a sensory neuropile in the auditory pathway of two groups of Orthoptera. J Comp Neurol 275:201-215. CrossRef Medline

Römer H, Lang A, Hartbauer M (2010) The signaller's dilemma: a costbenefit analysis of public and private communication. PLoS One 5:e13325. CrossRef Medline

Schmidt AK, Römer H (2011) Solutions to the cocktail party problem in insects: selective filters, spatial release from masking and gain control in tropical crickets. PLoS One 6:e28593. CrossRef Medline

Schul J (1999) Neuronal basis for spectral song discrimination in the bushcricket Tettigonia cantans. J Comp Physiol A Neuroethol Sens Neural Behav Physiol 184:457-461. CrossRef

Schul J, Sheridan RA (2006) Auditory stream segregation in an insect. Neuroscience 138:1-4. CrossRef Medline

Schul J, Mayo AM, Triblehorn JD (2012) Auditory change detection by a single neuron in an insect. J Comp Physiol A Neuroethol Sens Neural Behav Physiol 198:695-704. CrossRef Medline

Schwartz JJ, Bee MA (2013) Anuran acoustic signal production in noisy environments. In: Animal communication and noise, animal signals and communication, Vol 2 (Brumm H, ed), pp 91-132. Berlin: Springer.

Siegert ME, Römer H, Hartbauer M (2013) Maintaining acoustic communication at a cocktail party: heterospecific masking noise improves signal detection through frequency separation. J Exp Biol 216:4655-4665. CrossRef Medline

Sismondo E (1990) Synchronous, alternating, and phase-locked stridulation by a tropical katydid. Science 249:55-58. CrossRef Medline

Stolting H, Stumpner A (1998) Tonotopic organization of auditory receptors of the bushcricket Pholidoptera griseoaptera (Tettigoniidae, Decticinae). Cell Tissue Res 294:377-386. CrossRef Medline

Strauß J, Lehmann GU, Lehmann AW, Lakes-Harlan R (2012) Spatial organization of tettigoniid auditory receptors: insights from neuronal tracing. J Morphol 273:1280-1290. CrossRef Medline

Stumpner A (1996) Tonotopic organization of the hearing organ in a bushcricket. Naturwissenschaften 83:81-84. CrossRef

Stumpner A (1997) An auditory interneurone tuned to the male song frequency in the duetting bushcricket Ancistrura nigrovittata (Orthoptera, Phaneropteridae). J Exp Biol 200:1089-1101. Medline

Stumpner A (1998) Picrotoxin eliminates frequency selectivity of an auditory interneuron in a bushcricket. J Neurophysiol 79:2408-2415. Medline

Stumpner A, Molina J (2006) Diversity of intersegmental auditory neurons in a bushcricket. J Comp Physiol A Neuroethol Sens Neural Behav Physiol 192:1359-1376. CrossRef Medline

Triblehorn JD, Schul J (2013) Dendritic mechanisms contribute to stimulus-specific adaptation in an insect neuron. J Neurophysiol 110: 2217-2226. CrossRef Medline

Wehner R (1989) Matched filters-neural models of the external world. J Comp Physiol A Neuroethol Sens Neural Behav Physiol 161:511-531. CrossRef

Wiley RH (2006) Signal detection and animal communication. Adv Study Behav36:217-247.

Wiley RH (2013) Signal detection, noise, and the evolution of communication. In: Animal communication and noise, animal signals and communication, Vol 2 (Brumm H, ed), pp 7-30. Berlin: Springer.

Wohlers DW, Huber F (1982) Processing of sound signals by six types of neurons in the prothoracic ganglion of the cricket, Gryllus campestris L. J Comp Physiol A Neuroethol Sens Neural Behav Physiol 146:161-173. CrossRef

Yost WA (1997) The cocktail party problem: 40 years later. In: Binaural and spatial hearing in real and virtual environments (Gilkey RH, Anderson, TR, eds.), pp 329-347. Mahwah, NJ: Erlbaum. 